\title{
Statins enhance expression of growth factors and activate the PI3K/Akt-mediated signaling pathway after experimental intracerebral hemorrhage*
}

\author{
Dongmei Yang ${ }^{1}$, Yuxia Han ${ }^{1}$, Jianfeng Zhang ${ }^{1}$, Michael Chopp ${ }^{2,3}$, Donald M. Seyfried ${ }^{1 \#}$ \\ ${ }^{1}$ Departments of Neurosurgery, Henry Ford Health System, Detroit, USA \\ ${ }^{2}$ Departments of Neurology, Henry Ford Health System, Detroit, USA \\ ${ }^{3}$ Department of Physics, Oakland University, Rochester, USA

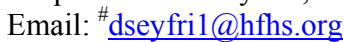

Received 21 January 2012; revised 11 February 2012; accepted 9 March 2012

\begin{abstract}
Previous studies have demonstrated that statins improve neurological outcome and promote neurovascular recovery after ICH. This study is designed to examine whether simvastatin and atorvastatin affect levels of growth factors and activate the Akt signaling pathway during the recovery phase after intracerebral hemorrhage (ICH) in rats. Sixty (60) male Wistar rats were subjected to ICH by stereotactic injection of $100 \mu \mathrm{L}$ of autologous blood into the striatum and were treated with or without simvastatin or atorvastatin. Neurological functional outcome was evaluated by behavioral tests (mNSS and corner turn test) at different time points after ICH. Brain extracts were utilized for Enzyme-linked immunosorbent assay (ELISA) analyses to measure vascular endothelial growth factor (VEGF); brain-derived neurotrophin factor (BDNF) expression, and nerve growth factor (NGF). Western blot was used to measure the changes in the Akt-mediated signaling pathway. Both the simvastatin- and atorvastatin-treated animals had significant neurological improvement at 2 weeks post-ICH. Simvastatin and atorvastatin treatment increased the expression of BDNF, VEGF and NGF in both lowand high-dose groups at 7 days after ICH $(p<0.05)$. Phosphorylation of Akt, glycogen synthase kinase- $3 \beta$ (GSK-3 $\beta$ ), and cAMP response element-binding proteins (CREB) were also increased at 7 days after statin treatment. These results suggest that the therapeutic effects of statins after experimental ICH may be mediated by the transient induction of BDNF, VEGF and NGF expression and the activation of the Aktmediated signaling pathway.
\end{abstract}

\footnotetext{
*Sources of Funding: Supported by National Institute of Health grant RO1 NS058581-01A1 (DMS).

${ }^{\#}$ Corresponding author.
}

Keywords: Statin; Growth Factor; Experimental; Intracerebral Hemorrhage

\section{INTRODUCTION}

The statins are a class of medications that lower cholesterol levels by inhibiting the 3-hydroxy-3-methylglutaryl-coenzyme A (HMG-CoA) reductase enzyme that controls the rate of cholesterol production in the body [13]. Statins are among the most commonly prescribed drugs for patients with heart disease, because they reduce cardiac events in patients with high cholesterol levels and with increased risk for heart disease [1]. Recent experimental investigations indicate that statins also exert several other beneficial effects independent of their cholesterol-reducing properties [3]. In animal models, statins improve neurological recovery after ischemic stroke, traumatic brain injury (TBI), and intracerebral hemorrhage (ICH) [4-7].

Brain-derived neurotrophin factors (BDNF), vascular endothelial growth factor (VEGF) and nerve growth factor (NGF) are structurally related polypeptides that have a fundamental role in neuronal and vascular development in the brain at both the embryonic and developmental stages [8-10]. The beneficial effects of statins after stroke and TBI may be attributed to increased expression of cerebral BDNF and VEGF in the ischemic and injury boundary zone $[7,11,12]$. The therapeutic effects of statin treatments may also be mediated by endothelial NO synthase (eNOS), the phosphatidylinositol-3 kinase (PI3K)/ Akt signaling pathway, upregulated B-cell leukemia/lymphoma 2 (Bcl-2) mRNA and protein expression, and augmented heat shock proteins in the context of brain injury caused by stroke and trauma [7,13-17].

In previous studies performed on ICH animal models we found that atorvastatin and simvastatin, which are initiated from $24 \mathrm{~h}$ post-ICH and then sequentially for 7 days 
at a dose of $2 \mathrm{mg} / \mathrm{kg}$, improve neurological functional outcome and promote neurovascular recovery 4 weeks after ICH $[5,18,19]$. Little is known, however, about the underlying mechanisms that mediate the neurorestorative effects of statins in ICH, but one hypothesis is that similar signal pathways may be utilized after ICH as in brain injury models initiated by stroke and TBI. In this study, we investigated the possible molecular mechanisms of atorvastatin and simvastatin treatment after $\mathrm{ICH}$, and postulate that activation of the PI3K/Akt signaling pathway and expression of VEGF, BDNF and NGF precede neurological recovery in this experimental $\mathrm{ICH}$ model. We also examined whether statins administered within the critical first $24 \mathrm{~h}$ post-ICH have a therapeutic effect.

\section{MATERIALS AND METHODS}

\subsection{Experimental Model}

All experimental procedures were approved by the Institutional Animal Care and Use Committee (IACUC No. 1061) at Henry Ford Health System. Sixty (60) adult male Wistar rats were subjected to ICH by stereotactic infusion of $100 \mu \mathrm{L}$ autologous whole blood into the striatal region adjacent to the subventricular zone (SVZ). Sham rats underwent identical surgery excepting whole blood infusion. After the operation, the animals were divided randomly into four groups: 1) sham group $(\mathrm{n}=6)$; 2) saline control group $(\mathrm{n}=18)-12 \mathrm{ICH}$ rats received saline orally at 1 day post-ICH and consecutively for 7 days, and six ICH rats received saline orally at 3 and $24 \mathrm{~h}$ after $\mathrm{ICH} ; 3)$ simvastatin-treated group $(\mathrm{n}=18)-12 \mathrm{ICH}$ rats received $2 \mathrm{mg} / \mathrm{kg}$ simvastatin orally daily at 1 day post$\mathrm{ICH}$ and consecutively for 7 days, and six ICH rats received $20 \mathrm{mg} / \mathrm{kg}$ simvastatin orally at 3 and $24 \mathrm{~h}$ after $\mathrm{ICH}$; and 4) atorvastatin-treated group $(\mathrm{n}=18)-12 \mathrm{ICH}$ rats received $2 \mathrm{mg} / \mathrm{kg}$ atorvastatin orally daily at 1 day post-ICH and consecutively for 7 days, and six ICH rats were given $20 \mathrm{mg} / \mathrm{kg}$ atorvastatin orally at 3 and $24 \mathrm{~h}$ after ICH. Six rats from the low-dose simvastatin- or atorvastatin-treated group and 6 rats from the corresponding saline control group were selected and sacrificed at 14 days after $\mathrm{ICH}$. The other 42 rats were sacrificed at 7 days. The choice of the dose and time points was based on previous published studies $[5,20]$.

\subsection{Neurobehavioral Testing}

Functional outcome was assessed using the modified neurological severity score (mNSS) [21] and the corner turn test [22] at 1, 4, 7 and 14 days after injury. The mNSS is used to assess neurological functions by a $0-18$ composite score of motor, sensory, balance, and reflex measures, with higher scores implying greater neuronlogical injury. The corner turn test measures long-term functional recovery by counting the number of times that an animal turns toward the non-impaired, ipsilateral (left) side when placed in a corner. The non-injured rat randomly turns equally either left or right.

\subsection{Enzyme-Linked Immunosorbent Assay}

Brain tissues of control and statin-treated animals were obtained from the boundary zone around the hematoma (i.e., bregma $-1 \mathrm{~mm}$ to $1 \mathrm{~mm}$, border region encompassing the hematoma) at 7 and 14 days after ICH. The samples were homogenized in cold RIPA lysis buffer kept at $4^{\circ} \mathrm{C}$ for $10 \mathrm{~min}$ and centrifuged for $10 \mathrm{~min}$ at $10,000 \mathrm{~g}$ at $4^{\circ} \mathrm{C}$. The lysis buffer contains $20 \mathrm{mM}$ Tris $\mathrm{pH} 7.6,100$ $\mathrm{mM} \mathrm{NaCl}, 1 \%$ Nonidet P-40, $0.1 \%$ SDS, 1\% deoxycholic acid, 10\% glycerol, $1 \mathrm{mM}$ EDTA, $1 \mathrm{mM} \mathrm{NaVO}$, $50 \mathrm{mM} \mathrm{NaF}$, and cocktail I of protease inhibitors (Calbiochem, San Diego, CA). The protein concentration of each cleared lysate sample was determined by bicinchoninic acid (BCA) protein assay (Pierce, Rockford, IL). Using ELISA kits (R\&D Systems, Minneapolis, MN), NGF, BDNF, and VEGF protein levels were measured with equal amounts of cleared lysates from brain tissues collected at different time points. ELISA was carried out using the Titertek Multiskan MCC/340 equipment (Labsystems, Helsinki, Finland).

\subsection{Western Blots}

To measure the levels of Akt, GSK3 and CREB, equal amounts of the samples were treated with SDS sample buffer and loaded on $10 \%$ or $14 \%$ SDS-polyacrylamide gels. The resolved proteins were electro-transferred to a PVDF membrane and incubated for $1 \mathrm{~h}$ at room temperature in Blocking Solution [5\% nonfat dried milk dissolved in TBST buffer, $\mathrm{pH}$ 7.5 (10 mM Tris_HCl_150 $\mathrm{mM} \mathrm{NaCl} 0.1 \%$ Tween 20)]. The filters were probed overnight at $4^{\circ} \mathrm{C}$ in $1 \%$ blocking solution in TBST containing primary antibodies. The primary antibodies were a 1:2000 dilution of the antibody against Akt and phospho-Akt (serine-473), a 1:1000 dilution of the antibody against GSK3 $\beta$ and phospho-GSK3 $\beta$ (serine-9) (Cell Signaling Technology, Beverly, MA), and a 1:1000 dilution of the antibody against $\beta$-actin (Santa Cruz Biotechnology, Santa Cruz, CA). Membranes were washed three times with TBST buffer and incubated with secondary antibodies (1:2500; Jackson Immunological Research Laboratory, West Grove, PN) for $1 \mathrm{~h}$, followed by washing four times. Signal detection was performed with an enhanced chemiluminescence kit (Pierce, Rock-ford, IL) and quantitated by using a GS-700.

\subsection{Statistical Analysis}

Statistical analyses of the results of the ELISA and Western blotting between statin-treated and control groups 
were evaluated using ANOVA techniques. Data are reported as mean \pm standard error of the mean (SEM). Statistical significance was inferred at $p \leq 0.05$. All measurements were performed by observers blinded to individual treatments.

\section{RESULTS}

\subsection{Statins Improve Functional Recovery}

All ICH rats displayed similar and marked neurological impairments compared to the sham-operated groups at day 1 after ICH. No significant differences were found between treatment groups and control group prior to 1 week post-ICH. Improvement of mNSS was significant at 2 weeks post-ICH in the statin-treated groups compared to the control group (Figure 1). Similarly, cornering scores for the simvastatin- and atorvastatin-treated groups improved significantly at 2 weeks post-ICH compared to the control group (Figure 1); however, the simvastatin group appeared to improve somewhat more than the atorvastatin group, but the amount was not statistically significant. Furthermore, there were no significant differences between the low-dose $(2 \mathrm{mg} / \mathrm{kg})$ and high-dose $(20$ $\mathrm{mg} / \mathrm{kg}$ ) treated groups in both functional tests at 1 week after $\mathrm{ICH}$.

\subsection{Statins Increase BDNF, VEGF and NGF Expression}

To measure how statins may affect the level of growth
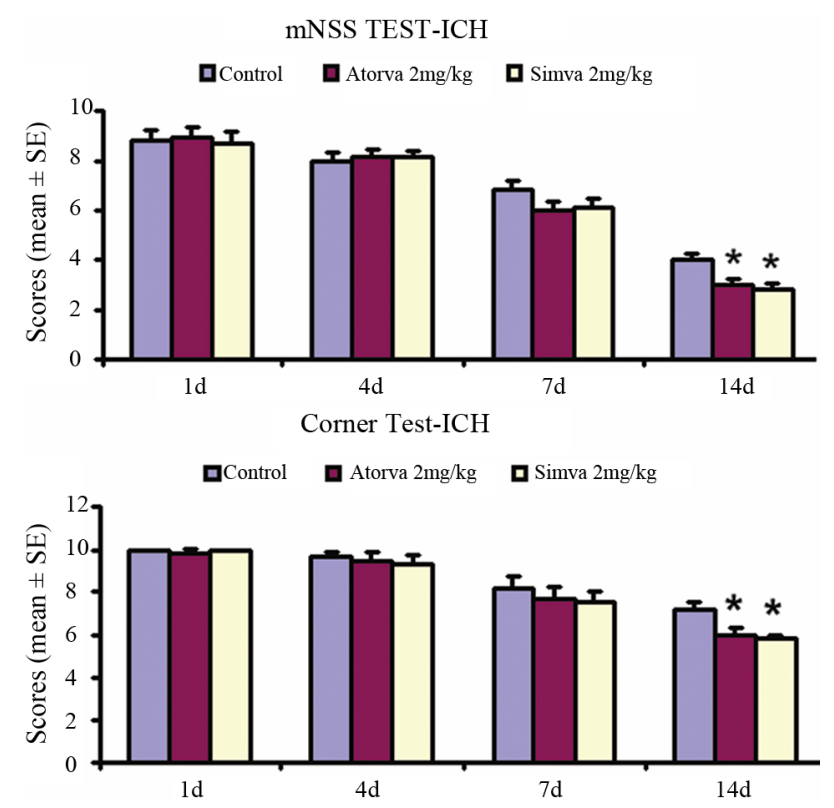

Figure 1. Quantitative bar graphs show results of mNSS and corner turn test of the control and treatment groups at day 14 after ICH. Statins improved neurological outcome after ICH. Statistical significance level is: ${ }^{*} p \leq 0.05$ versus the saline-treated group. factors after $\mathrm{ICH}$, the protein levels of BDNF, VEGF and NGF were studied using ELISA at 7 and 14 days after ICH. BDNF, VEGF and NGF levels in statin-treated animals at 7 days post-ICH were significantly enhanced ( $p$ $<0.01$ ) when compared to the corresponding control animals (Figure 2). The low-dose statins given for 7 days showed no significantly different expression levels of these neurotrophic and vascular factors, when compared with the high-dose statins given acutely. Consistent with the results of functional tests, no significant differences in growth factor expression were found between the simvastatin and atorvastatin groups. At 14 days postICH, expression levels of BDNF, VEGF and NGF were decreased in the statin-treated animals, and there was no significant difference when compared with the control group (Figure 2).

\subsection{Statins Activate Akt, GSK3, and CREB}

To determine the possible mechanism by which statins promote neurological functional recovery PI3K/Akt pathway, one of the most widely studied signal pathways, was analyzed by Western blot analysis. The results revealed that both simvastatin and atorvastatin significantly enhanced the phosphorylation of Akt at day 7 after ICH (Figure 3). The phosphorylation of GSK3, the downstream signaling target of the Akt pathway, was increased 2-fold in the lesion boundary area of both statin-treated groups at day 7 after ICH (Figure 3). Because one of the downstream targets of Akt is CREB, we further examined the levels of total CREB and phosphorylated CREB in total tissue lysates to confirm that CREB is involved in statin-mediated neurological recovery after ICH. As expected, the level of p-CREB-S133 is also elevated at day 7 after treatment with statins (Figure 3). There were no significant differences between the simvastatin- and atorvastatin-treated groups or between acute high-dose treatment and early time period treatment.

\section{DISCUSSION}

It has been found that activation of Akt and upregulation of growth factor expression by atorvastatin may contribute to neurologic recovery after experimental ischemic stroke and brain trauma $[7,23]$. The expression of growth factors and the activation of the PI3K/Akt signaling pathway by statins in ICH have not been established. In the present study, we demonstrate for the first time that simvastatin and atorvastatin increase BDNF, VEGF and NGF expression, and activate this signaling pathway and improve neurological function in an autologous ICH model. This finding is a significant step toward understanding statins' mechanism of action and the possible benefits of applying them after ICH. 

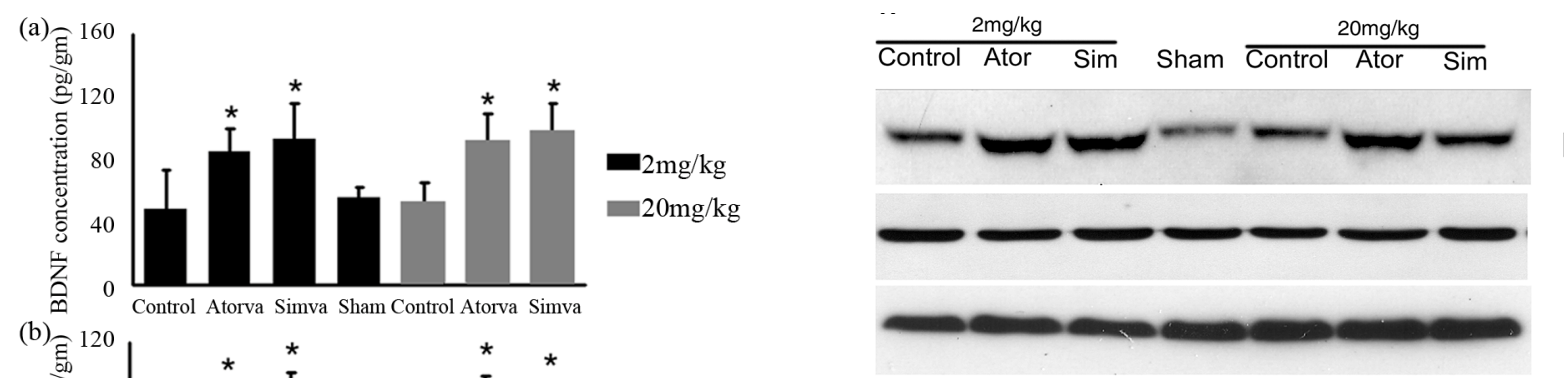

P-Akt

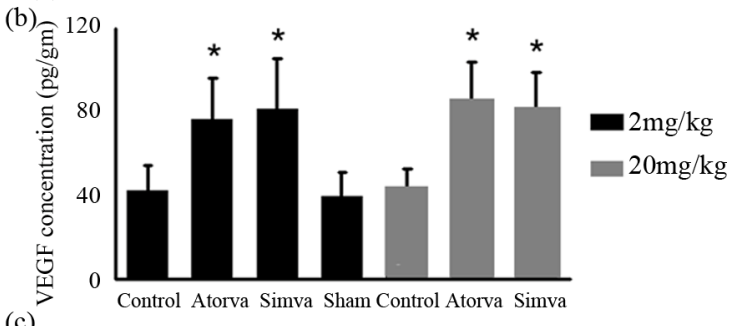

(a)

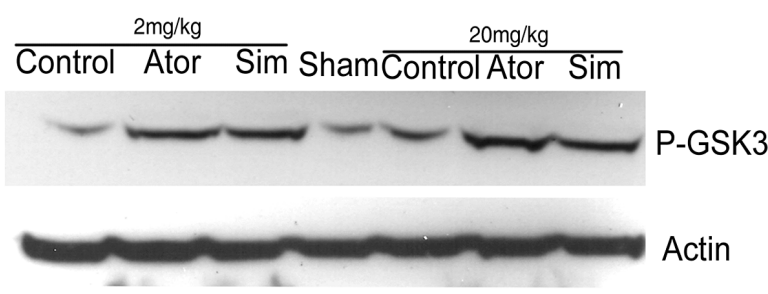

(b)

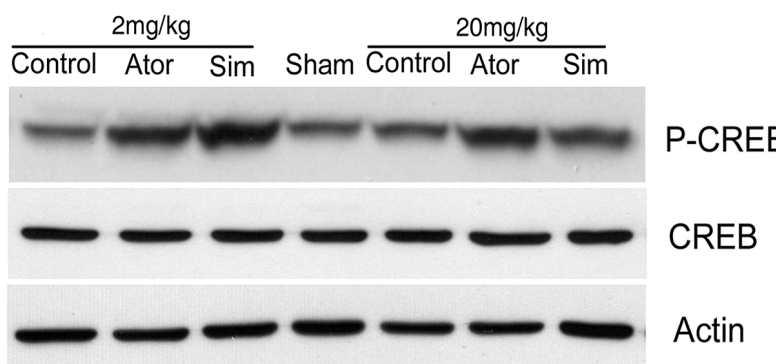

(c)
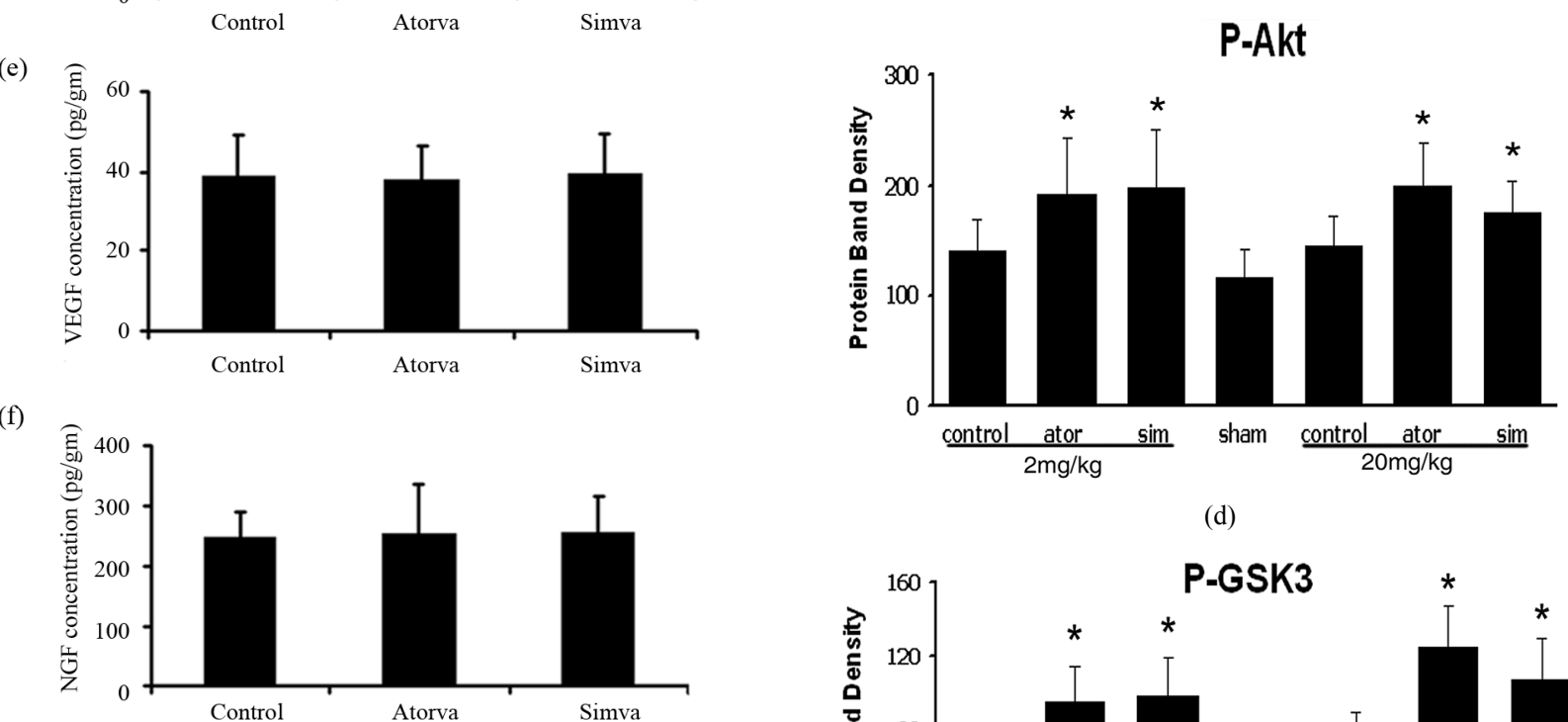

Figure 2. Bar graphs demonstrate enzyme-linked immunosorbent assay (ELISA) expression of (a) and (d) brain-derived neurontrophin factor (BDNF), (b) and (e) vascular endothelial growth factor (VEGF) and (c) and (f) nerve growth factor (NGF) in the boundary area around rat hematoma after atorvastatin, simvastatin or saline treatment at 7 days (a, b, and c) and 14 days (d, e, and f) after ICH. ${ }^{*} p \leq 0.05$ versus the saline-treated group.

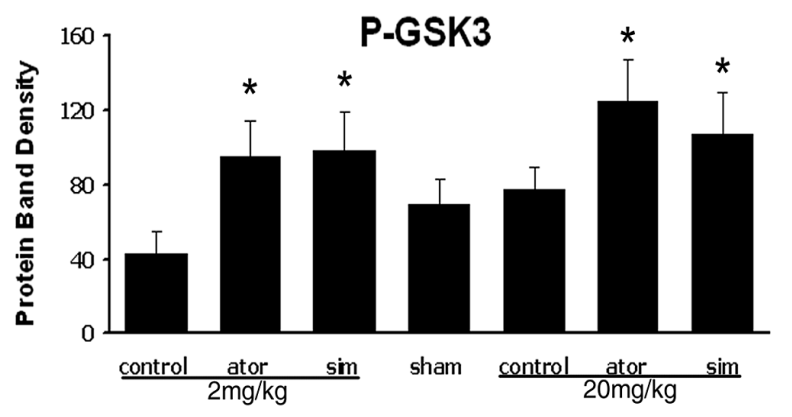

(e) 


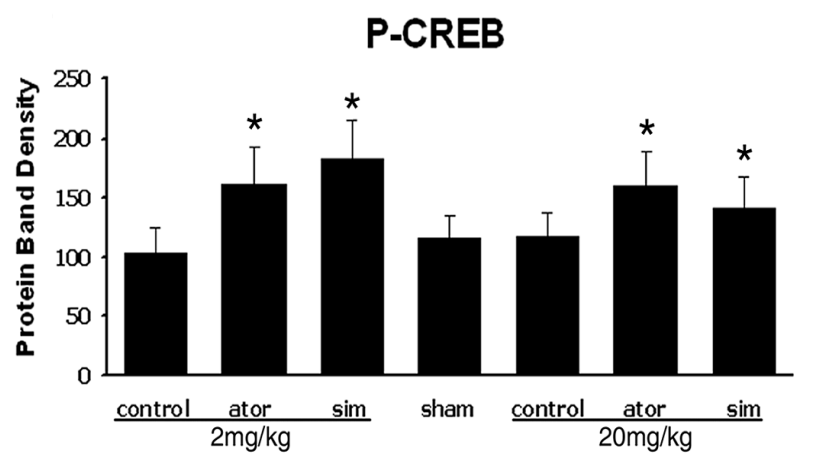

(f)

Figure 3. Representative Western blot analysis shows the expression of phosphorylated and total Akt (a), phosphorylated GSK3 (b), and phosphorylated and total CREB (c) from the brain tissue around rat hematoma treated with atorvastatin, simvastatin or saline at 7 days after ICH. Bar graphs show densitometry measurement of phosphorylated Akt (d), phosphorylated GSK3 (e), and phosphorylated CREB (f) from each group. Data in the bar graphs are represented as mean \pm SE. ${ }^{*} p \leq 0.05$ as compared with the saline-treated group.

BDNF and NGF are two important neurotrophic factors that induce the survival, development, and function of neurons [24]. After cerebral ischemia, BDNF and NGF mRNAs increase in the hippocampal neuron [25], and treatment with external NGF can ameliorate delayed neuronal death [26]. VEGF promotes angiogenesis and vasculogenesis and stimulates neurogenesis and axonal outgrowth $[27,28]$. Treatment of experimental ischemic stroke with atorvastatin reduced neurologic deficits and upregulated VEGF and BDNF and induced angiogenesis, neurogenesis, and synaptic plasticity [12]. Our previous results demonstrate that statins can promote angiogenesis and neuronal plasticity after ICH $[5,19]$. We propose that the statin-induced increase of these growth factors may enhance neuronal and synaptic plasticity, which improves functional recovery. Data from the present study support this hypothesis and are also consistent with our observations in trauma and ischemic stroke injury models. The level of growth factors may be related to neurological functional recovery after ICH but temporally may not always be consistent with it. Our study showed that BDNF, NGF and VEGF increased 2- to 3-fold at 7 days post-ICH compared with control and were back to the control level at 14 days post-ICH; however, the significant functional recovery was observed from 14 days. This may suggest that neurorecovery and tissue restoration is initiated by increased expression of growth factors and that a lag time may occur before ultimate functional result is observed.

The PI3K/Akt signaling pathway is commonly activated in diverse cellular processes, including cellular proliferation and survival [29]. This signaling pathway can be activated by neurotrophic factors and has been implicated in the survival of neuronal cells and reduction of neurological disorders [9,29]. Statins rapidly and effectively promote the activation of Akt by phosphorylation of Akt in vitro and in experimental models of ischemic stroke and TBI [23,29,30]. Statin-induced capillary-like tube formation is inhibited by a neutralizing antibody against VEGFR2 and inhibition of PI3K. NGF can protect against oxidative stress in a PI3K-dependent manner [6,17]. All these observations are consistent with the fact that Akt is expressed at high levels during neurogenesis [31]. In this study we demonstrate for the first time that statins stimulate the PI3K/Akt cascade in the setting of ICH.

GSK-3 is one of the primary downstream targets of PI3K/Akt cell survival pathway [32,33]. GSK-3 signaling plays a vital role in the proliferation and differentiation of progenitor cells during brain development [34]. CREB is a well-established transcription factor downstream of neurotrophins [35]. CREB is also known to be a major target of PI 3-kinase/Akt/GSK-3 signaling pathway [34]. CREB regulation of neural precursor survival involves expression of BDNF that provides a growth environment for the residential precursor cells after focal cerebral ischemia, ICH and other brain injuries [7,13]. In parallel, our data show that statins increase phosphorylated Akt, which is associated with the increase of phosphorylated GSK-3. Statins also increase phosphorylation of CREB in the hematoma boundary area after $\mathrm{ICH}$, a phenomenon that suggests transcription activation mediated by the active CREB form; however, it is unknown whether the upregulation of BDNF, NGF and VEGF observed in this study resulted from activation of Akt or was a direct effect of the statins. The phosphorylation of GSK-3 exhibits a temporal pattern similar to that of CREB, whereas the expression of phospho-Akt precedes that of GSK-3. These similar patterns suggest that the phosphorylation of GSK-3 is associated with the phosphorylation of Akt and CREB. GSK-3 inactivation by Akt-induced phosphorylation may enhance CREB transcriptional activity by abrogating the inhibitory effect of GSK-3, thereby mediating the neuroprotective effect of statins.

In our previous study, atorvastatin significantly reduced the extent of neurological injury at a dose of $2 \mathrm{mg} / \mathrm{kg} /$ day for 7 days, but not at higher doses $(8 \mathrm{mg} / \mathrm{kg} /$ day for 7 days) after experimental ICH [5]. Consistent with those results, the $2 \mathrm{mg} / \mathrm{kg}$ dose used in this study improved neurological functional recovery. Statins administered at a dose of $20 \mathrm{mg} / \mathrm{kg}$ given twice acutely (within $24 \mathrm{~h}$ ) provided effects similar to that of the low-dose groups. Therefore, high-dose statins are effective in this model if given acutely, but not if administered during the entire first week after $\mathrm{ICH}$ (i.e., $8 \mathrm{mg} / \mathrm{kg}$ ) [5]. No secondary hemorrhage was observed in either group. Although sim- 
vastatin and atorvastatin are different due to their intrinsic differences in plasma life [36] and permeability across the BBB [37], with simvastatin having greater lipophilic properties, our study demonstrated that both simvastatin and atorvastatin induced similar growth factor expression and activation of neurological benefits post-ICH.

\section{CONCLUSION}

Simvastatin and atorvastatin demonstrate therapeutic potential acutely after treatment of $\mathrm{ICH}$, as seen in this experimental model. These beneficial effects may be partially mediated by induction of BDNF, VEGF and NGF expression, and by activation of the PI3K/Akt signaling pathway.

\section{ACKNOWLEDGEMENTS}

Special thanks to Susan MacPhee-Gray for editorial assistance.

\section{REFERENCES}

[1] Lardizabal, J.A., and Deedwania, P. (2011) Lipid-lowering therapy with statins for the primary and secondary prevention of cardiovascular disease. Cardiology Clinics, 29, 87-103. doi:10.1016/i.ccl.2010.10.002

[2] Megnien, J.L. and Simon, A. (1998) Therapeutic management of hypercholesterolemia. La Presse Médicale, 27, 2056-2061.

[3] Takemoto, M. and Liao, J.K. (2001) Pleiotropic effects of 3-hydroxy-3-methylglutaryl coenzyme a reductase inhibitors. Arteriosclerosis, Thrombosis, and Vascular Biology, 21, 1712-1719. doi:10.1161/hq1101.098486

[4] Wible, E.F. and Laskowitz, D.T. (2010) Statins in traumatic brain injury. Neurotherapeutics, 7, 62-73. doi:10.1016/j.nurt.2009.11.003

[5] Seyfried, D., Han, Y., Lu, D., Chen, J., Bydon, A. and Chopp, M. (2004) Improvement in neurological outcome after administration of atorvastatin following experimental intracerebral hemorrhage in rats. Journal of Neurosurgery, 101, 104-107. doi:10.3171/jns.2004.101.1.0104

[6] Chen, J., Zhang, Z.G., Li, Y., Wang, Y., Wang, L., Jiang, H., Zhang, C., Lu, M., Katakowski, M., Feldkamp, C.S. and Chopp, M. (2003) Statins induce angiogenesis, neurogenesis and synaptogenesis after stroke. Annals of Neurology, 53, 743-751. doi:10.1002/ana.10555

[7] Wu, H., Lu, D., Jiang, H., Xiong, Y., Qu, C., Li, B., Mahmood, A., Zhou, D. and Chopp, M. (2008) Simvastatin-mediated upregulation of VEGF and BDNF, activation of the PI3K/Akt pathway and increase of neurogenesis are associated with therapeutic improvement after traumatic brain injury. Journal of Neurotrauma, 25, 130-139. doi:10.1089/neu.2007.0369

[8] Hempstead, B.L. (2006) Dissecting the diverse actions of pro- and mature neurotrophins. Current Alzheimer Research, 3, 19-24. doi:10.2174/156720506775697061

[9] Reichardt, L.F. (2006) Neurotrophin-regulated signalling pathways. Philosophical Transactions of the Royal Society B: Biological Sciences, 361, 154-1564.

doi:10.1098/rstb.2006.1894

[10] Zhang, Z.G., Zhang, L., Jiang, Q., Zhang, R., Davies, K., Powers, C., Bruggen, N. and Chopp, M. (2000) VEGF enhances angiogenesis and promotes blood-brain barrier leakage in the ischemic brain. Journal of Clinical Investigation, 106, 829-838. doi:10.1172/JCI9369

[11] Chen, J., Zacharek, A., Li, A., Zhang, C., Ding, J., Roberts, C., Lu, M., Kapke, A. and Chopp, M. (2006) Vascular endothelial growth factor mediates atorvastatin-induced mammalian achaete-scute homologue-1 gene expression and neuronal differentiation after stroke in retired breeder rats. Neuroscience, 141, 737-744. doi:10.1016/j.neuroscience.2006.04.042

[12] Chen, J., Zhang, C., Jiang, H., Li, Y., Zhang, L., Robin, A., Katakowski, M., Lu, M. and Chopp, M. (2005) Atorvastatin induction of VEGF and BDNF promotes brain plasticity after stroke in mice. Journal of Cerebral Blood Flow \& Metabolism, 25, 281-290. doi:10.1038/sj.jcbfm.9600034

[13] Chen, X.N., Xu, J., Feng, Z., Fan, M., Han, J.Y. and Yang, Z. (2010) Simvastatin combined with nifedipine enhances endothelial cell protection by inhibiting ROS generation and activating Akt phosphorylation. Acta Pharmacologica Sinica, 31, 813-820. doi:10.1038/aps.2010.58

[14] Ito, D., Ito, O., Mori, N., Muroya, Y., Cao, P.Y., Takashima, K., Kanazawa, M. and Kohzuki, M. (2010) Atorvastatin upregulates nitric oxide synthases with Rhokinase inhibition and Akt activation in the kidney of spontaneously hypertensive rats. Journal of Hypertension, 28, 2278-2288. doi:10.1097/HJH.0b013e32833e0924

[15] Johnson-Anuna, L.N., Eckert, G.P., Franke, C., Igbavboa, U., Muller, W.E. and Wood, W.G. (2007) Simvastatin protects neurons from cytotoxicity by up-regulating Bcl-2 mRNA and protein. Journal of Neurosurgery, 101, 77-86. doi:10.1111/j.1471-4159.2006.04375.x

[16] Kretz, A., Schmeer, C., Tausch, S. and Isenmann, S. (2006) Simvastatin promotes heat shock protein 27 expression and Akt activation in the rat retina and protects axotomized retinal ganglion cells in vivo. Neurobiology of Disease, 21, 421-430. doi:10.1016/j.nbd.2005.08.003

[17] Rahbek, U.L., Dissing, S., Thomassen, C., Hansen, A.J. and Tritsaris, K. (2005) Nerve growth factor activates aorta endothelial cells causing PI3K/Akt- and ERK-dependent migration. Pflügers Archiv European Journal of Physiology, 450, 355-361. doi:10.1007/s00424-005-1436-0

[18] Karki, K., Knight, R.A., Han, Y., Yang, D., Zhang, J., Ledbetter, K.A., Chopp, M. and Seyfried, D.M. (2009) Simvastatin and atorvastatin improve neurological outcome after experimental intracerebral hemorrhage. Stroke, 40, 3384-3389. doi:10.1161/STROKEAHA.108.544395

[19] Yang, D., Knight, R.A., Han, Y., Karki, K., Zhang, J., Ding, C., Chopp, M. and Seyfried, D.M. (2011) Vascular recovery promoted by atorvastatin and simvastatin after experimental intracerebral hemorrhage: Magnetic resonance imaging and histological study. Journal of Neuro- 
surgery, 114, 1135-1142. doi:10.3171/2010.7.JNS10163

[20] Endres, M., Laufs, U., Huang, Z., Nakamura, T., Huang, P., Moskowitz, M.A. and Liao, J.K. (1998) Stroke protection by 3-hydroxy-3-methylglutaryl (HMG)-CoA reductase inhibitors mediated by endothelial nitric oxide synthase. Proceedings of the National Academy of Sciences of the United States of America, 95, 8880-8885. doi:10.1073/pnas.95.15.8880

[21] Chen, J., Sanberg, P.R., Li, Y., Wang, L., Lu, M., Willing, A.E., Sanchez-Ramos, J. and Chopp, M. (2001) Intravenous administration of human umbilical cord blood reduces behavioral deficits after stroke in rats. Stroke, 32, 2682-2688. doi:10.1161/hs1101.098367

[22] Zhang, L., Schallert, T., Zhang, Z.G., Jiang, Q., Arniego, P., Li, Q., Lu, M. and Chopp, M. (2002) A test for detecting long-term sensorimotor dysfunction in the mouse after focal cerebral ischemia. Journal of Neuroscience Methods, 117, 207-214. doi:10.1016/S0165-0270(02)00114-0

[23] Zhang, L., Zhang, Z.G., Liu, X.S., Hozeska-Solgot, A. and Chopp, M. (2007) The PI3K/Akt pathway mediates the neuroprotective effect of atorvastatin in extending thrombolytic therapy after embolic stroke in the rat. Arteriosclerosis, Thrombosis, and Vascular Biology, 27, 2470-2475. doi:10.1161/ATVBAHA.107.150748

[24] Huang, E.J. and Reichardt, L.F. (2001) Neurotrophins: roles in neuronal development and function. Annual Review of Neuroscience, 24, 677-736. doi:10.1146/annurev.neuro.24.1.677

[25] Lindvall, O., Ernfors, P., Bengzon, J., Kokaia, Z., Smith, M.L., Siesjo, B.K. and Persson, H. (1992) Differential regulation of mRNAs for nerve growth factor, brain-derived neurotrophic factor and neurotrophin 3 in the adult rat brain following cerebral ischemia and hypoglycemic coma. Proceedings of the National Academy of Sciences of the United States of America, 89, 648-652. doi:10.1073/pnas.89.2.648

[26] Shigeno, T., Mima, T., Takakura, K., Graham, D.I., Kato, G., Hashimoto, Y. and Furukawa, S. (1991) Amelioration of delayed neuronal death in the hippocampus by nerve growth factor. Journal of Neuroscience, 11, 2914-2919.

[27] Hess, C., Vuong, V., Hegyi, I., Riesterer, O., Wood, J., Fabbro, D., Glanzmann, C., Bodis, S. and Pruschy, M. (2001) Effect of VEGF receptor inhibitor PTK787/ ZK222584 [correction of ZK222548] combined with ionizing radiation on endothelial cells and tumour growth. British Journal of Cancer, 85, 2010-2016. doi:10.1054/bjoc.2001.2166
[28] Sondell, M., Lundborg, G. and Kanje, M. (1999) Vascular endothelial growth factor has neurotrophic activity and stimulates axonal outgrowth, enhancing cell survival and Schwann cell proliferation in the peripheral nervous system. Journal of Neuroscience, 19, 5731-5740.

[29] Wang, L., Gang Zhang, Z., Lan Zhang, R. and Chopp, M. (2005) Activation of the PI3-K/Akt pathway mediates cGMP enhanced-neurogenesis in the adult progenitor cells derived from the subventricular zone. Journal of Cerebral Blood Flow \& Metabolism, 25, 1150-1158. doi:10.1038/sj.jcbfm.9600112

[30] Carloni, S., Girelli, S., Buonocore, G., Longini, M. and Balduini, W. (2009) Simvastatin acutely reduces ischemic brain damage in the immature rat via Akt and CREB activation. Experimental Neurology, 220, 82-89. doi:10.1016/j.expneurol.2009.07.026

[31] Vojtek, A.B., Taylor, J., DeRuiter, S.L., Yu, J.Y., Figueroa, C., Kwok, R.P. and Turner, D.L. (2003) Akt regulates basic helix-loop-helix transcription factor-coactivator complex formation and activity during neuronal differentiation. Molecular and Cell Biology, 23, 44174427. doi:10.1128/MCB.23.13.4417-4427.2003

[32] Goold, R.G. and Gordon-Weeks, P.R. (2004) Glycogen synthase kinase 3 beta and the regulation of axon growth. Biochemical Society Transactions, 32, 809-811. doi:10.1042/BST0320809

[33] Kim, W.Y., Wang, X., Wu, Y., Doble, B.W., Patel, S., Woodgett, J.R. and Snider, W.D. (2009) GSK-3 is a master regulator of neural progenitor homeostasis. Nature Neuroscience, 12, 1390-1397. doi:10.1038/nn.2408

[34] Hur, E.M. and Zhou, F.Q. (2010) GSK3 signalling in neural development. Nature Reviews Neuroscience, 11, 539-551. doi: $10.1038 / \mathrm{nrn} 2870$

[35] Bender, R.A., Lauterborn, J.C., Gall, C.M., Cariaga, W. and Baram, T.Z. (2001) Enhanced CREB phosphorylation in immature dentate gyrus granule cells precedes neurotrophin expression and indicates a specific role of CREB in granule cell differentiation. European Journal of Neuroscience, 13, 679-686. doi:10.1046/j.1460-9568.2001.01432.x

[36] Endres, M. and Laufs, U. (2004) Effects of statins on endothelium and signaling mechanisms. Stroke, 35, 2708-2711. doi:10.1161/01.STR.0000143319.73503.38

[37] Zacco, A., Togo, J., Spence, K., Ellis, A., Lloyd, D., Furlong, S. and Piser, T. (2003) 3-hydroxy-3-methylglutaryl coenzyme A reductase inhibitors protect cortical neurons from excitotoxicity. Journal of Neuroscience, 23, 11104 11111. 